\title{
PCR Amplification and Transcription for Site-Specific Labeling of Large RNA Molecules by a Two-Unnatural-Base-Pair System
}

\author{
Michiko Kimoto, ${ }^{1,2}$ Rie Yamashige, ${ }^{1}$ Shigeyuki Yokoyama, ${ }^{1,3}$ and Ichiro Hirao ${ }^{1,2}$ \\ ${ }^{1}$ RIKEN Systems and Structural Biology Center (SSBC), 1-7-22 Suehiro-cho, Tsurumi-ku, Kanagawa, Yokohama, 230-0045, Japan \\ ${ }^{2}$ TAGCyx Biotechnologies, 1-6-126 Suehiro-cho, Tsurumi-ku, Kanagawa, Yokohama, 230-0045, Japan \\ ${ }^{3}$ Department of Biophysics and Biochemistry, Graduate School of Science, The University of Tokyo, 7-3-1 Hongo, Bunkyo-ku, \\ Tokyo 113-0033, Japan \\ Correspondence should be addressed to Ichiro Hirao, ihirao@riken.jp
}

Received 1 March 2012; Accepted 16 April 2012

Academic Editor: Subhendu Sekhar Bag

Copyright () 2012 Michiko Kimoto et al. This is an open access article distributed under the Creative Commons Attribution License, which permits unrestricted use, distribution, and reproduction in any medium, provided the original work is properly cited.

\begin{abstract}
For the site-specific labeling and modification of RNA by genetic alphabet expansion, we developed a PCR and transcription system using two hydrophobic unnatural base pairs: 7-(2-thienyl)-imidazo[4,5-b]pyridine (Ds) and 2-nitro-4-propynylpyrrole (Px) as a third pair for PCR amplification and Ds and pyrrole-2-carbaldehyde (Pa) for the incorporation of functional components as modified Pa bases into RNA by T7 transcription. To prepare Ds-containing DNA templates with long chains, the Ds-Px pair was utilized in a fusion PCR method, by which we demonstrated the synthesis of 282-bp DNA templates containing Ds at specific positions. Using these Ds-containing DNA templates and a biotin-linked Pa substrate (Biotin-PaTP) as a modified Pa base, 260mer RNA transcripts containing Biotin-Pa at a specific position were generated by T7 RNA polymerase. This two-unnatural-basepair system, combining the Ds-Px and Ds-Pa pairs with modified Pa substrates, provides a powerful tool for the site-specific labeling and modification of desired positions in large RNA molecules.
\end{abstract}

\section{Introduction}

Site-specific labeling and modification of large RNA molecules provide a wide variety of applications in many areas, such as biochemical and biophysical studies, synthetic biology, in vitro evolution, generation of functional nucleic acids, and construction of nanomaterials and biosensors. The sitespecific incorporation of functional nucleotide analogs into RNA molecules is performed by chemical synthesis, posttranscriptional modification, and enzymatic incorporation of nucleotide analogs as substrates. Among them, chemical synthesis is a commonly employed method. However, it is only capable of synthesizing small RNA molecules. Other site-specific modifications of RNA, by posttranscriptional modification [1,2] and enzymatic incorporation using cap or triphosphate analogs [3-6], are limited to terminal modifications of RNA. In contrast to these RNA labeling methods, introducing an artificial, extra base pair (unnatural base pair), as a third base pair, to in vitro transcription systems allows the site-specific incorporation of an unnatural base linked with functional groups into desired positions of RNA during transcription mediated by the unnatural base pair. Thus, several unnatural base pairs that function in polymerase reactions have rapidly been developed for sitespecific labeling of RNA molecules [7-18].

Here, for the site-specific incorporation of functional components into large RNA molecules, we report a fusion PCR and transcription system that employs two unnatural base pairs of 7-(2-thienyl)-imidazo [4,5-b] pyridine (Ds) and 2-nitro-4-propynylpyrrole $(\mathbf{P x})[19,20]$ and Ds and pyrrole2-carbaldehyde (Pa) [17] (Figure 1). The Ds-Px pair exhibits high efficiency and selectivity in PCR amplification as a third base pair. Under optimized conditions, more than $97 \%$ of the Ds-Px pair survives in the $10^{28}$-fold amplified DNAs through exponential 100-cycle PCR (10 cycles repeated 10 times). In particular, a modified Px base, 4-(4,5-dihydroxypent-1yn-1-yl)-2-nitropyrrole (Diol1-Px, Figure 1), has extremely high specificity as a pairing partner of Ds, and thus the misincorporation rates of Diol1-dPxTP and dDsTP opposite the natural bases in templates during PCR amplification 
Ds-Px pair for PCR amplification
Ds-Pa' pair for DNA sequencing
Ds-Pa pair

for T7 transcription
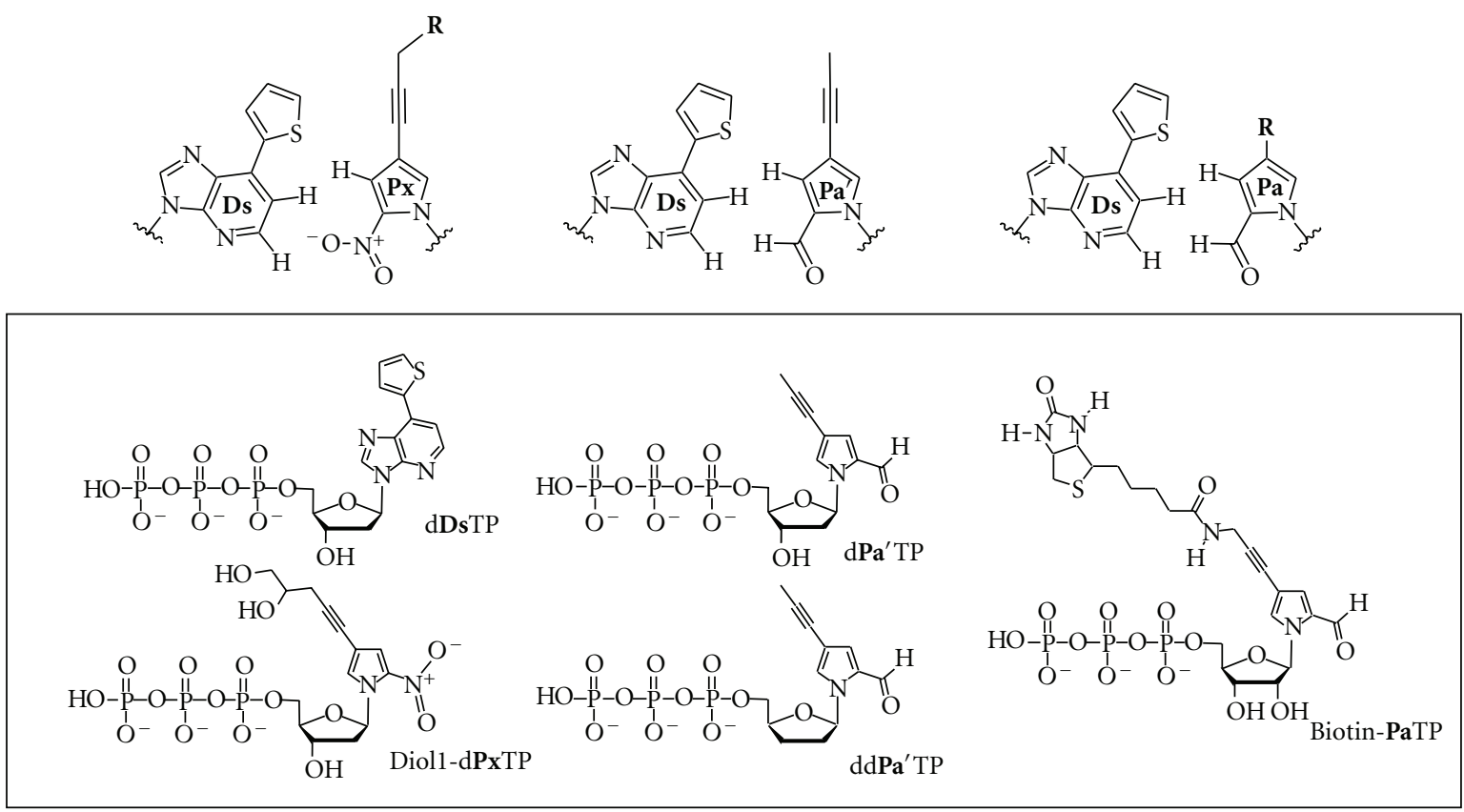

Figure 1: Chemical structures of the Ds-Px, Ds-Pa', and Ds-Pa pairs. The Ds-Px pair is employed for PCR amplification to prepare the Ds-containing DNA templates. The Ds-Pa' pair is employed for DNA sequencing, to determine the Ds position in the DNA templates. The Ds-Pa pair is employed for T7 transcription, to incorporate Biotin-Pa into RNA opposite Ds in the templates. Px and Pa bases modified with various functional groups (R) can also be used as substrates for PCR and transcription, respectively. The unnatural substrates used in this study are summarized in the box.

are as low as $0.005 \%$ per base pair per replication [20]. Here, we applied the Ds-Px pair to fusion PCR [21], using small DNA templates containing Ds with Diol1-dPxTP and dDsTP, to prepare large Ds-containing DNA templates for a genetic-expansion transcription system, and generated 282bp double-stranded DNA fragments containing the Ds and Diol1-Px pair. Using the Ds-containing DNA templates, we performed the site-specific incorporation of BiotinPaTP, as a functional unnatural base substrate, into 260mer transcripts by T7 RNA polymerase. In transcription, the $\mathbf{P a}$ base is superior to the Px base in terms of both the incorporation selectivity opposite Ds and the chemical stability of the nucleotide. However, in PCR amplification, the Ds-Px pair is more selective and efficient than the Ds-Pa pair, and thus to utilize both pairs' advantages, we developed a two-unnatural-base-pair system, the Ds-Px pair for fusion PCR, and the Ds-Pa pair for T7 transcription (Figure 2). By attaching functional groups of interest to the Pa base, this fusion PCR and transcription system could be a powerful tool for the site-specific labeling and functionalization of large RNA molecules.

\section{Materials and Methods}

2.1. Materials. Oligonucleotides containing Ds were synthesized with an Applied Biosystems 392 DNA synthesizer, using CE phosphoramidite reagents for the natural and Ds bases (Glen Research), and were purified by gel electrophoresis. Oligonucleotides comprising natural bases only were synthesized as described above or purchased from Invitrogen. AccuPrime Pfx DNA polymerase, SYBR Gold nucleic acid gel stain, and 10x PBS were purchased from Invitrogen. Streptavidin and silica-membrane columns for PCR product purification (Wizard SV Gel and PCR Clean-Up System) were purchased from Promega. T7 RNA polymerase was purchased from Takara. Toluidine Blue O was purchased from Chroma Gesellschaft Schmidt \& Co. The RNA ladder marker (DynaMarker, RNA Low II) was purchased from BioDynamics Laboratory, Inc. The DNA ladder marker (2-Log DNA Ladder) was purchased from New England Biolabs. Unnatural nucleoside triphosphates (dDsTP, Diol1-dPxTP, Biotin-PaTP, ddPa'TP, and dPa'TP) were synthesized as described previously $[17,19,20]$. The BigDye Terminator v1.1 Cycle Sequencing Kit was purchased from Applied Biosystems. Centri-Sep spin columns were purchased from Princeton Separations. Natural NTP and dNTP Sets (100 mM solutions: ATP, CTP, GTP, and UTP, and dATP, dCTP, dGTP and dTTP, resp.) were purchased from GE Healthcare. Gel images were analyzed with a bioimaging analyzer, LAS4000 (Fuji Film). The plasmid DNA used for PCR amplification was provided by Dr. Tsutomu Kishi (RIKEN).

2.2. Preparation of DNA Templates for T7 Transcription. To prepare the 282-bp double-stranded DNA fragments containing the $\mathbf{D s}-\mathbf{P x}$ pair as templates for T7 transcription, 


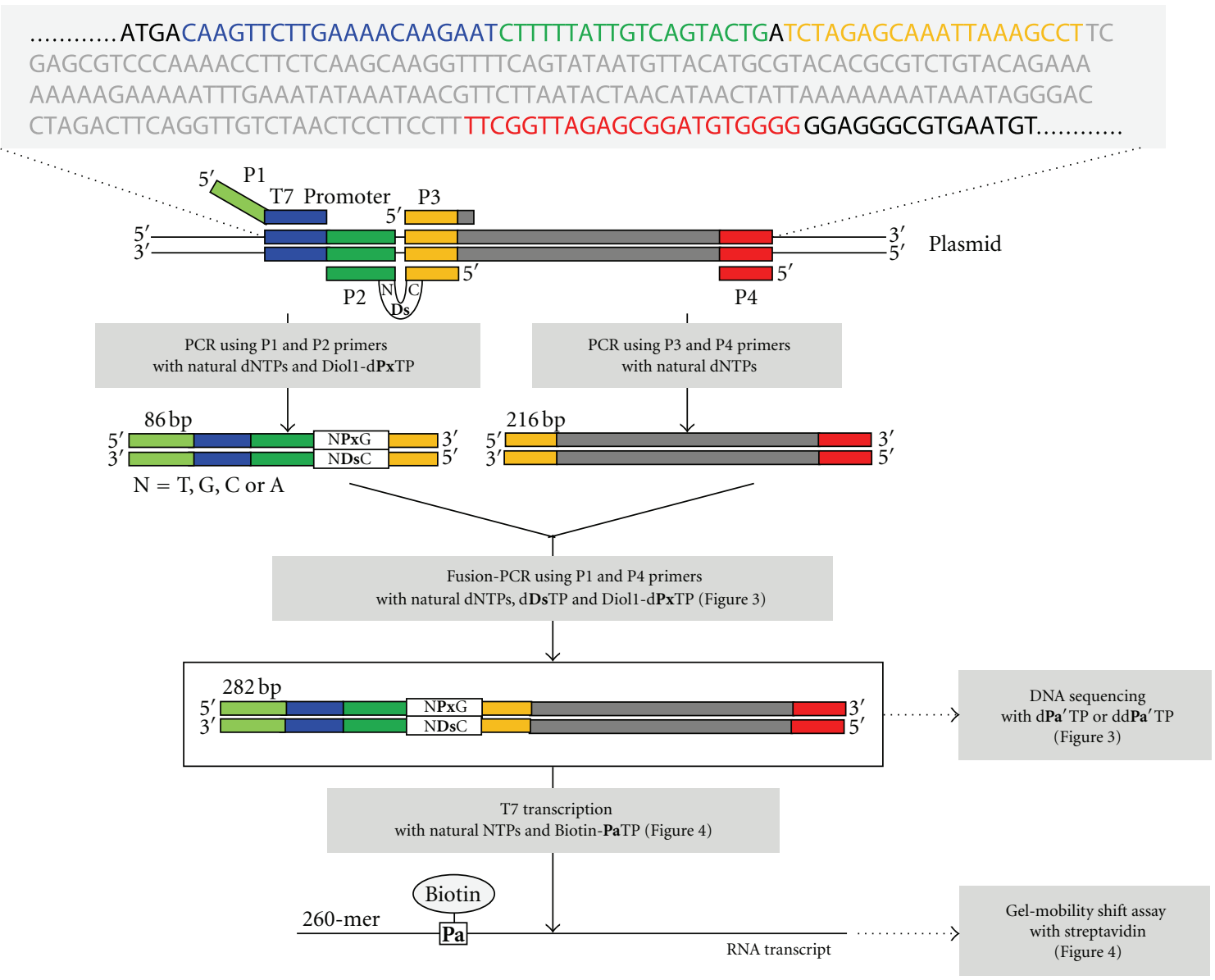

FIGURE 2: Experimental scheme of PCR amplification and transcription by a two-unnatural-base-pair system for site-specific biotin labeling of RNA molecules. DNA fragments containing the Ds-Px pair at the internal positions were prepared by a fusion PCR method. The original plasmid sequence, used as the PCR template, is shown at the top. The CDsN sequence $(N=T, G, A$, or $C)$ in the P2 primer is integrated into the 86-bp DNA fragments. The 282-bp products generated by fusion PCR were used as templates for T7 transcription with Biotin-PaTP.

two steps of PCR using AccuPrime Pfx DNA polymerase were performed as follows. First, 86-bp DNA fragments containing the Ds-Diol1-Px pair were amplified through 25-cycle PCR $(50 \mu \mathrm{L})$ from $1 \mathrm{ng} / \mu \mathrm{L}$ plasmid DNA template, by using $1 \mu \mathrm{M}$ 5'-primer (P1: 5'-AAGCTTAATACGACT CACTATAGCAAGTTCTTGAAAACAAGAAT- $3^{\prime}$, the T7 promoter region is italicized, and the region complementary to the plasmid is underlined) and $3^{\prime}$-primer (P2: 5'-AGGCTT TAATTTGCTCTAGACDsNCAGTACTGACAATAAAAAG-3', $\mathrm{N}=\mathrm{T}, \mathrm{G}, \mathrm{A}$, and $\mathrm{C}$; the region complementary to the plasmid is underlined) and $0.02 \mathrm{U} / \mu \mathrm{L}$ AccuPrime Pfx DNA polymerase, in the manufacturer's reaction buffer with $600 \mu \mathrm{M}$ each natural dNTP, $50 \mu \mathrm{M}$ Diol1-dPxTP, and $2 \mathrm{mM}$ $\mathrm{MgSO}_{4}$. The final concentrations of dNTPs and $\mathrm{MgSO}_{4}$ were adjusted by adding $300 \mu \mathrm{M}$ each natural dNTP, $50 \mu \mathrm{M}$ Diol1$\mathrm{dPxTP}$, and $1 \mathrm{mM} \mathrm{MgSO}$ to the $1 \mathrm{xAccuPrime} P f x$ reaction mix, which originally contained $300 \mu \mathrm{M}$ each natural dNTP and $1 \mathrm{mM} \mathrm{MgSO}_{4}$ [20]. PCR conditions were $30 \mathrm{sec}$ at $94^{\circ} \mathrm{C}$, $30 \mathrm{sec}$ at $55^{\circ} \mathrm{C}$, and $2 \mathrm{~min}$ at $65^{\circ} \mathrm{C}$ per cycle, and the amplified PCR products were purified by gel electrophoresis. As control experiments, we also performed PCR amplification using the P1 and P2 primers without Ds ( $5^{\prime}$-AGGCTT TAATTTGCTCTAGACATCAGTACTGACAATAAAAAG-3'), in the presence and absence of Diol1-dPxTP. A 216-bp double-stranded DNA fragment was amplified by 25cycle PCR using $1 \mu \mathrm{M}$ P3 primer (5'-TCTAGAGCAAAT TAAAGCCTTCG-3' ${ }^{\prime}$, the sequence complementary to the P2 primer is underlined) and $\mathrm{P} 4$ primer $\left(5^{\prime}\right.$-CCCCACATCCGC TCTAACCGAA-3') from $1 \mathrm{ng} / \mu \mathrm{L}$ plasmid DNA template in the reaction buffer, containing $600 \mu \mathrm{M}$ each natural dNTP and $2 \mathrm{mM} \mathrm{MgSO}_{4}$, followed by gel purification. Next, we performed fusion PCR $(100 \mu \mathrm{L})$ with AccuPrime Pfx DNA polymerase, using $1 \mu \mathrm{M}$ each of the $\mathrm{P} 1$ and $\mathrm{P} 4$ primers, and the purified 86-bp (25 nM) and 216-bp (1 nM) DNA fragments, in the reaction buffer with $600 \mu \mathrm{M}$ each natural dNTP and $2 \mathrm{mM} \mathrm{MgSO}_{4}$, in the presence or absence of $50 \mu \mathrm{M}$ each of $\mathrm{dDsTP}$ and Dioll-dPxTP. PCR conditions were $30 \mathrm{sec}$ at $94^{\circ} \mathrm{C}$ and $3 \mathrm{~min}$ at $65^{\circ} \mathrm{C}$ per cycle. After 25-cycle PCR amplification, the products were purified by passage through silica-membrane columns, according to the manufacturer's instructions. The concentrations of the purified products were determined from their UV 
absorbance. The purified products were used for DNA sequencing and transcription experiments.

2.3. DNA Sequencing. The cycle sequencing reaction $(10 \mu \mathrm{L})$ was performed with the Cycle Sequencing mix $(4 \mu \mathrm{L})$ from the BigDye Terminator v1.1. Cycle Sequencing Kit, $1 \mu \mathrm{L}$ of $2 \mu \mathrm{M}$ Sequencing primer (5'-TGACAAGTTCTT GAAAACAAGAAT- $3^{\prime}$ ), $2 \mu \mathrm{L}$ of $250 \mu \mathrm{M} \mathrm{dPa} \mathbf{A}^{\prime}$ TP or ddPa' ${ }^{\prime}$ TP, and $3 \mu \mathrm{L}$ of $50 \mathrm{nM}$ DNA fragments [20]. The sequencing cycle parameters were 25 cycles of $10 \mathrm{sec}$ at $96^{\circ} \mathrm{C}, 5 \mathrm{sec}$ at $50^{\circ} \mathrm{C}$, and $4 \mathrm{~min}$ at $60^{\circ} \mathrm{C}$. The residual dye terminators were removed with Centri-Sep columns, and the solutions were dried with a centrifugal vacuum evaporator. The residues were resuspended in $3 \mu \mathrm{L}$ of a formamide/BlueDextran/ EDTA loading buffer and analyzed with an ABI 377 DNA sequencer, using a $6 \%$ polyacrylamide- $6 \mathrm{M}$ urea gel. The sequence peak patterns were analyzed with the Applied Biosystems PRISM sequencing analysis software, v3.2.

2.4. Transcription. Transcription $(20 \mu \mathrm{L})$ was performed in a buffer containing $40 \mathrm{mM}$ Tris- $\mathrm{HCl}$ ( $\mathrm{pH} 8.0$ ), $24 \mathrm{mM} \mathrm{MgCl}_{2}$, $5 \mathrm{mM}$ DTT, $2 \mathrm{mM}$ spermidine, and $0.01 \%$ Triton X-100, in the presence of $2 \mathrm{mM}$ each NTP, 0 or $2 \mathrm{mM}$ BiotinPaTP, 100 nM 282-bp DNA template, and $2.5 \mathrm{U} / \mu \mathrm{L}$ T7 RNA polymerase. After an incubation at $37^{\circ} \mathrm{C}$ for $3 \mathrm{~h}$, the reaction was quenched by adding an equivalent volume of a denaturing solution, containing $10 \mathrm{M}$ urea in 1xTBE. The reaction mixtures were heated at $75^{\circ} \mathrm{C}$ for $3 \mathrm{~min}$, and $7.5 \mu \mathrm{L}$ aliquots were fractionated on a $5 \%$ denaturing polyacrylamide- $7 \mathrm{M}$ urea gel. After electrophoresis, the transcribed products on the gel were stained with toluidine blue and detected by a LAS 4000 imager. For gel-mobility shift assays, the full-length products were purified on a $5 \%$ denaturing polyacrylamide$7 \mathrm{M}$ urea gel.

2.5. Gel-Mobility Shift Assay. We detected the biotinylated RNA transcripts (260-mer) by gel-mobility shift assays, using streptavidin. We incubated the mixture $(10 \mu \mathrm{L})$ of $0.5 \mathrm{pmol}$ transcripts and excess amounts of streptavidin (800 ng) for $1 \mathrm{~h}$ at $25^{\circ} \mathrm{C}$, in $1 \mathrm{x}$ PBS containing $5 \%$ glycerol. The biotinylated RNA-streptavidin complexes were separated from the free RNAs on a non-denaturing $8 \%$ polyacrylamide gel, and the RNAs on the gel were stained with SYBR Gold and detected by an LAS 4000 imager.

\section{Results and Discussion}

3.1. Fusion-PCR Mediated by the Ds-Px Pair for Preparing Long DNA Templates Containing Ds at Desired Positions. To examine fusion PCR [21] using the Ds-Px pair, we prepared the 282-bp double-stranded DNA (dsDNA) templates containing the Ds and Diol1-Px pair at internal positions by two PCR steps, using four PCR primers (P1 to P4) and a plasmid DNA, as shown in Figure 2. The P2 primer contains Ds and the P3 region. In the first PCR, we used the plasmid as an initial PCR template to introduce the Ds-Px pair, and prepared two dsDNA fragments (86- and 216-bp) by using AccuPrime Pfx DNA polymerase [20]. The 86-bp DNA fragment was amplified by the $\mathrm{P} 1$ and $\mathrm{P} 2$ primers and corresponds to the $5^{\prime}$ region of the 282-bp template, which contains the T7 promoter sequence in its $5^{\prime}$ region and the Ds-Px pair in its $3^{\prime}$ region. The 216-bp DNA fragment was amplified by the P3 and P4 primers and corresponds to the $3^{\prime}$ region of the 282-bp template, which comprises natural bases only. Since the P2 and P3 primers share a 20mer complementary sequence, the 282-bp templates can be prepared by the second PCR step (fusion PCR) using the 86bp and 216-bp DNA fragments and the P1 and P4 primers.

For preparing the Ds-containing 86-bp DNA fragments, we performed 25 cycles of PCR using each of the four P2 primers encoding a $5^{\prime}$-CDsN-3' sequence $(\mathrm{N}=\mathrm{T}, \mathrm{G}, \mathrm{C}$, or $\mathrm{A})$, in the presence of Diol1-dPxTP, as well as the natural dNTPs as substrates and the P1 primer. Each amplified PCR product was purified by gel electrophoresis, to completely remove the original plasmid template. As control experiments, we also performed PCR amplification using a non-Ds-containing $\mathrm{P} 2$ primer with a $5^{\prime}$-CAT- $3^{\prime}$ sequence, in the presence and absence of the unnatural base substrate, Dioll-dPxTP. The end-point analysis of the PCR products on the agarose gel revealed no differences among their PCR amplification efficiencies (data not shown). Thus, under the conditions employed here by using AccuPrime Pf $x$ DNA polymerase, the nature of the natural base nucleotide at the $5^{\prime}$-neighboring position of Ds does not affect the amplification efficiency.

To prepare the full-length 282-bp templates by fusion PCR, we performed 25-cycle PCR using the P1 and P4 primers and the gel-purified 86-bp and 216-bp DNA fragments as templates, in the presence of Diol1-dPxTP and dDsTP together with the natural dNTPs as substrates. Figure 3 shows the agarose gel of the PCR-amplified products. The fusion PCR involving the Ds-Px pair with all four sequence contexts generated the full-length products with high efficiency, similar to that of the control experiment using the DNA fragments with only the natural base sequence context.

The site-specific incorporation of the Ds-Px pair into each of the 282-bp DNA fragments was confirmed by dideoxy dye terminator sequencing of the Ds-containing DNA strands in the presence of the dideoxyribonucleoside or deoxyribonucleoside triphosphate of 4-propynylpyrrole2-carbaldehyde ( $\mathrm{dPa}^{\prime} \mathrm{TP}$ or $\mathrm{dd} \mathbf{P a}^{\prime} \mathrm{TP}$, Figure 1), another pairing partner of Ds (Figure 3), according to our previously reported method $[19,20]$. In the presence of $\mathrm{dd}_{\mathbf{P a}}{ }^{\prime} \mathrm{TP}$, the sequencing reaction terminated at the unnatural base position because of the incorporation of $\mathrm{ddPa}^{\prime} \mathrm{TP}$ opposite Ds in the DNA templates, and thus the following base peaks disappeared. In the sequencing in the presence of $\mathrm{dPa}^{\prime} \mathrm{TP}$, the $\mathrm{d} \mathbf{P a}^{\prime} \mathrm{TP}$ was incorporated opposite Ds in the DNA templates, and the following base peaks appeared, but there is no peak at the unnatural base position because no dye terminator corresponding to the unnatural base was present in the sequencing reaction. By comparing the sequence patterns of the DNA fragment containing $5^{\prime}-\mathrm{APxG}-3^{\prime}$ with that containing $5^{\prime}$-ATG-3', the differences are clearly observed. These results indicated that the Ds-Px pair functions well in fusion PCR using AccuPrime Pfx DNA polymerase, and from the sequencing analysis, the retention rate of the unnatural base pair in the amplified DNA fragments was more than $97 \%$. 


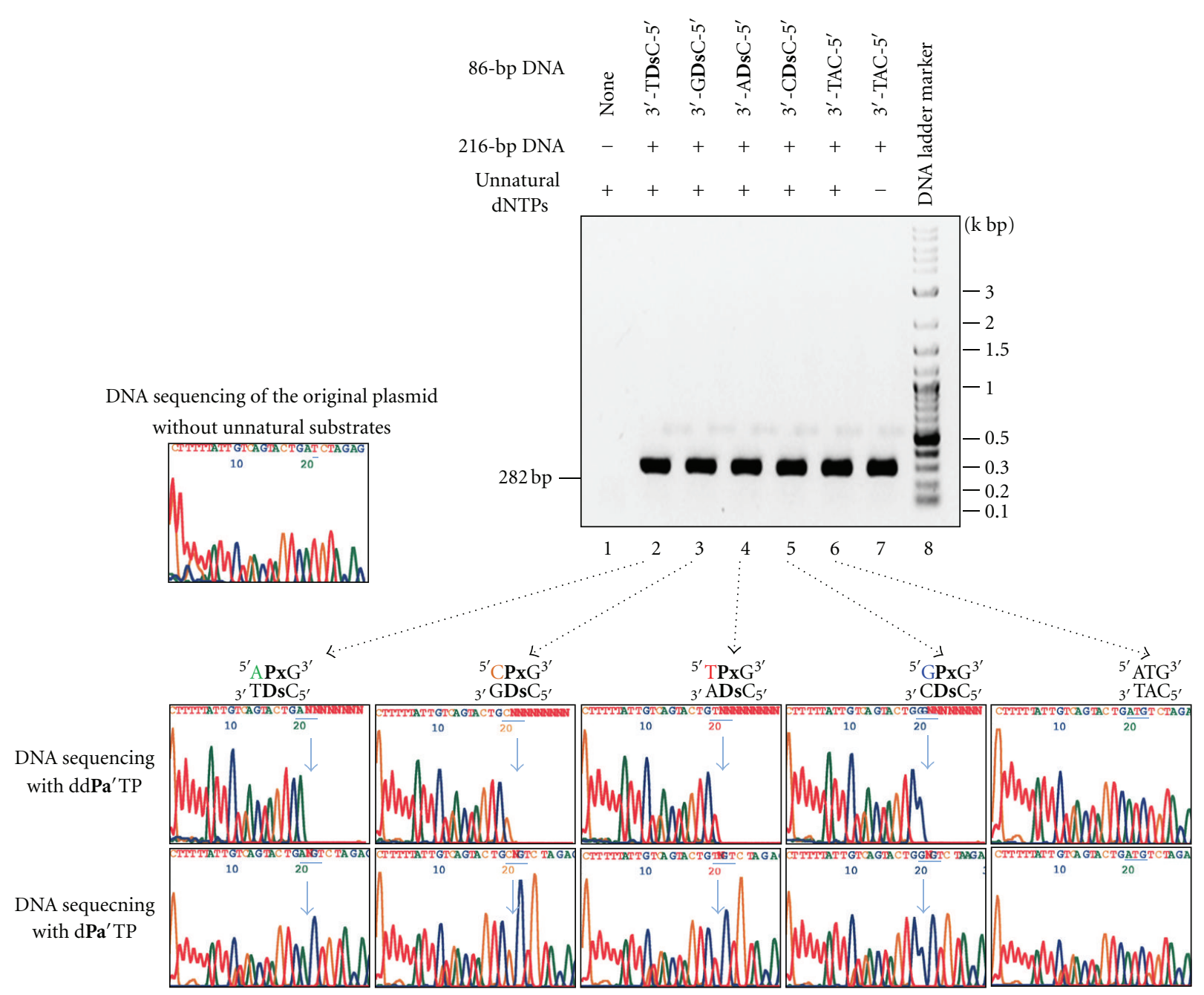

FIGURE 3: PCR amplification and DNA sequencing of 282-bp DNA templates containing Ds at specific positions. After 25-cycle fusion PCR using the P1 and P4 primers and the 86-bp and 216-bp DNA fragments as templates in the presence or absence of dDsTP and Diol1-dPxTP, the amplified PCR products were analyzed on a 1\% agarose gel. Using the purified PCR products, the sequencing reactions were performed in the presence of $\mathrm{dd} \mathbf{P a}^{\prime} \mathrm{TP}$ (upper sequencing panels) or $\mathrm{dPa}$ 'TP (lower sequencing panels). The light blue arrow indicates the unnatural base position. The light blue bar indicates the inserted sequence.

3.2. T7 Transcription, Mediated by the Ds-Pa Pair for the Site-Specific Biotinylation of Large RNA Molecules Using a Biotin-Pa Substrate. Next, we examined the transcription by T7 RNA polymerase, using the 282-bp DNA templates containing Ds and Biotin-PaTP as a functional component. Transcription was performed in the presence of $2 \mathrm{mM}$ Biotin-PaTP, as well as $2 \mathrm{mM}$ natural NTPs, at $37^{\circ} \mathrm{C}$ for $3 \mathrm{~h}$, and the full-length transcripts (260-mer) were analyzed by denaturing polyacrylamide gel electrophoresis (Figure 4). As control experiments, the non-Ds-containing DNA templates with the $5^{\prime}$-CAT- $3^{\prime}$ sequence, which were PCR amplified with or without the unnatural base substrates, were transcribed with or without Biotin-PaTP. All of the transcription reactions produced similar yields of the 260 -mer products.

To characterize the selectivity of the Biotin-Pa incorporation into RNA fragments by transcription, we performed gel-mobility shift assays of the biotinylated transcripts in the presence of streptavidin (Figure 4). As for the incorporation site, we already confirmed the site specificity of the Biotin$\mathrm{Pa}$ incorporation opposite Ds in templates with various sequence contexts $[17,18]$. From the gel shift assays, we estimated the biotinylation yields of each transcript (92\% of the transcript was biotinylated for the $3^{\prime}-\mathrm{CDsC}-5^{\prime}$ template sequence, $84 \%$ for $3^{\prime}$-TDsC- $5^{\prime}, 75 \%$ for $3^{\prime}$-GDsC$5^{\prime}$, and $72 \%$ for $\left.3^{\prime}-\mathrm{ADsC}-5^{\prime}\right)$. Thus, in contrast to fusion PCR involving the Ds-Px pair, the biotinylation selectivity depended to some extent on the neighboring bases of Ds, and the order of the effective sequence contexts of the 282bp DNA templates was the same as that of the previously determined effective sequence context of the 35-mer synthetic DNA template for a 17-mer RNA transcript with Pa [18]. The biotinylation yields resulted from the sum of the 25-cycle PCR and T7 transcription selectivities. However, the sequencing analysis (Figure 3) indicated that the retention of the unnatural-base-pair in the amplified DNA was more than 97\% after 25-cycle PCR, and thus the biotinylation yields 


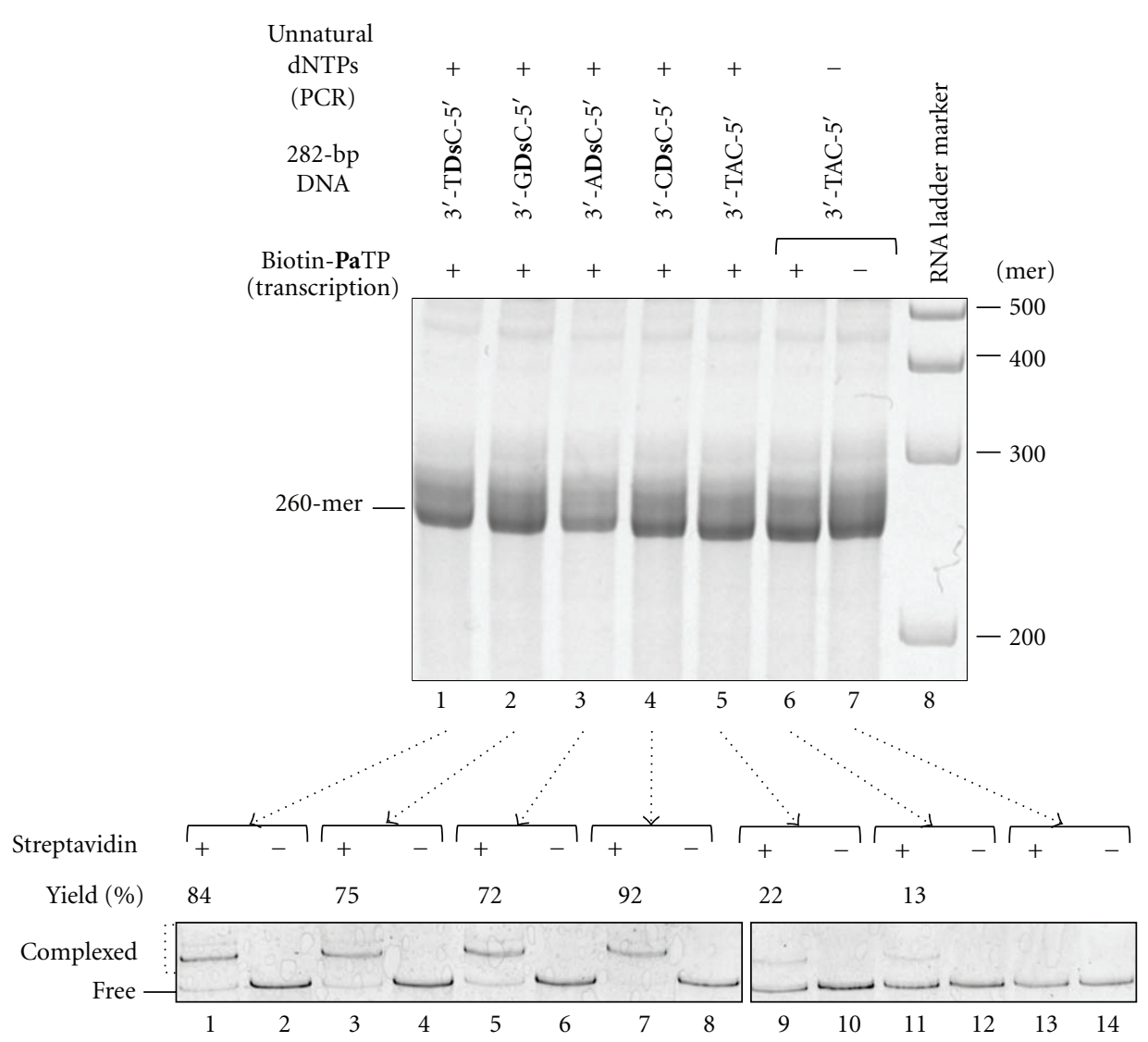

Figure 4: T7 transcription for site-specific biotin labeling of 260-mer transcripts. Transcripts from each 282-bp DNA template containing $3^{\prime}$-TDsC-5', $3^{\prime}-\mathrm{GDsC}-5^{\prime}, 3^{\prime}-\mathrm{ADsC}-5^{\prime}$, or $3^{\prime}-\mathrm{CDsC}-5^{\prime}$, in the presence of the natural NTPs $(2 \mathrm{mM})$ and Biotin-PaTP $(2 \mathrm{mM})$, were analyzed on a $5 \%$ denaturing polyacrylamide gel (upper gel image). After the purification of each 260-mer transcript, gel-mobility shift assays were performed using streptavidin. Biotinylated RNA-streptavidin complexes were separated from free RNAs on an 8\% nondenaturing polyacrylamide gel, and the amounts of the complexes (yields) were determined from the band intensities. The experiments were independently repeated twice, and representative data are shown. The gel-mobility shift values were averaged from two data sets.

actually depended on the transcription selectivities of the DsPa pair when using each DNA template.

We also assessed the misincorporation of Biotin-PaTP opposite the natural bases in the two-unnatural base pair system. The misincorporation rates of Biotin-PaTP into the transcript using the control $3^{\prime}$-TAC-5' templates, which were prepared by fusion PCR in the presence and absence of dDsTP and Diol1-dPxTP, were 22\% and 13\%, respectively. Thus, the misincorporations correspond to only $0.096 \%$ (for $22 \%$ total) and $0.054 \%$ (for $13 \%$ total) per position in the 260-mer transcript, as calculated by the following equation: $y=(1-x / 100)^{260}$, where $x \%$ is the Biotin-Pa misincorporation rate opposite the natural bases per position and $y$ is the ratio of nonbiotinylated transcript. These misincorporation rates were lower than those of the noncognate natural base pairings, as we previously reported that the misincorporation of biotin-linked UTP opposite G, C, and T in the T7 transcription of a non-A-containing template was around $0.16 \%$ per base [17]. In addition, the value of $0.054 \%$ is quite consistent with the value $(0.06 \%)$ that we previously determined by $\mathrm{T} 7$ transcription of 152-mer transcripts in the presence of $2 \mathrm{mM}$ Biotin-PaTP and $2 \mathrm{mM}$ natural NTPs
[17]. This misincorporation rate also corresponds to the sum of the 25-cycle PCR and T7 transcription selectivities. Thus, the difference between $0.096 \%$ and $0.054 \%$ was caused by the Ds misincorporation into the template strand during 25-cycle PCR amplification with dDsTP and Diol1dPxTP. In the 25-cycle fusion PCR, the PCR primers were mostly consumed, and the theoretical amplification cycle was estimated as ten $\left(2^{10} \approx 1000=\right.$ [primer] $/[216$-bp DNA fragments]). Therefore, the Ds misincorporation opposite a natural base per replication was calculated as approximately $0.004 \%(\approx(0.096 \%-0.054 \%) / 10)$. Consequently, the Ds misincorporation into the nontemplate strand would occur, and thus the misincorporation rates of Diol1-dPxTP and dDsTP opposite the natural bases in templates during PCR amplification would be $0.008 \%$, which is as high as that of the noncognate natural base pairings.

\section{Conclusion}

In this study, we demonstrated the two-unnatural-base-pair system for the site-specific labeling of large RNA molecules by fusion PCR and T7 transcription. In fusion PCR, more 
than $97 \%$ of the Ds-Px pair was retained in the amplified 282-bp DNA fragments, and the misincorporation rate of the unnatural bases opposite the natural bases was $0.008 \%$ per base per replication. We employed the natural base substrates at relatively high concentrations $(600 \mu \mathrm{M}$ each), as compared to the unnatural base substrates (dDsTP and Diol1-dPxTP, $50 \mu \mathrm{M}$ each) in the fusion PCR, to reduce the misincorporation rate. By using this method, the biotinlinked unnatural base was site-specifically incorporated at predetermined positions of RNA transcripts with selectivities ranging from $72 \%$ to $92 \%$, depending on the sequence contexts around the unnatural base. In addition, the misincorporation rate of the biotin-unnatural base opposite the natural bases was around $0.05 \%$ per natural base. These selectivity and misincorporation rates were obtained using $2 \mathrm{mM}$ unnatural and natural base substrates in T7 transcription. The incorporation selectivity $(90-96 \%)$ and the misincorporation rate of Biotin-Pa depend on the ratio of Biotin-PaTP to the natural base NTPs in transcription [17]. Thus, the incorporation efficiency and misincorporation rates can be adjusted by changing the concentration ratios between the unnatural and natural base substrates. To reduce the misincorporation of the unnatural base substrate opposite the natural bases, with the sacrifice of the incorporation efficiency of the unnatural base substrate at the desired positions, transcription should be performed with $1 \mathrm{mM}$ unnatural base substrate and $2 \mathrm{mM}$ natural base substrates $[12,17]$. Since several functional groups can be attached to the $\mathbf{P a}$ base, this method could be applied to a wide range of site-specific labeling and functionalization of large RNA molecules.

\section{Acknowledgments}

The authors thank Tsutomu Kishi for preparing the plasmid and helpful discussions and Akira Sato for preparing the unnatural substrates. This work was supported by the Targeted Proteins Research Program and the RIKEN Structural Genomics/Proteomics Initiative, the National Project on Protein Structural and Functional Analyses, Ministry of Education, Culture, Sports, Science and Technology of Japan, and by Grants-in-Aid for Scientific Research (KAKENHI 19201046 to I. H., 20710176 to M. K.) from the Ministry of Education, Culture, Sports, Science and Technology of Japan.

\section{References}

[1] P. Z. Qin and A. M. Pyle, "Site-specific labeling of RNA with fluorophores and other structural probes," Methods, vol. 18, no. 1, pp. 60-70, 1999.

[2] D. Chatterji and V. Gopal, "Fluorescence spectroscopy analysis of active and regulatory sites of RNA polymerase," Methods in Enzymology, vol. 274, pp. 456-478, 1996.

[3] F. Huang, G. Wang, T. Coleman, and N. Li, "Synthesis of adenosine derivatives as transcription initiators and preparation of $5^{\prime}$ fluorescein- and biotin-labeled RNA through onestep in vitro transcription," $R N A$, vol. 9, no. 12, pp. 1562-1570, 2003.
[4] A. Draganescu, S. C. Hodawadekar, K. R. Gee, and C. Brenner, "Fhit-nucleotide specificity probed with novel fluorescent and fluorogenic substrates," Journal of Biological Chemistry, vol. 275, no. 7, pp. 4555-4560, 2000.

[5] S. Seetharaman, M. Zivarts, N. Sudarsan, and R. R. Breaker, "Immobilized RNA switches for the analysis of complex chemical and biological mixtures," Nature Biotechnology, vol. 19, no. 4, pp. 336-341, 2001.

[6] L. Zhang, L. Sun, Z. Cui, R. L. Gottlieb, and B. Zhang, " 5 '-sulfhydryl-modified RNA: initiator synthesis, in vitro transcription, and enzymatic incorporation," Bioconjugate Chemistry, vol. 12, no. 6, pp. 939-948, 2001.

[7] Y. Tor and P. B. Dervan, "Site-specific enzymatic incorporation of an unnatural base, N6-(6-ammohexyl)isoguanosine, into RNA," Journal of the American Chemical Society, vol. 115, no. 11, pp. 4461-4467, 1993.

[8] I. Hirao, T. Ohtsuki, T. Fujiwara et al., "An unnatural base pair for incorporating amino acid analogs into proteins," Nature Biotechnology, vol. 20, no. 2, pp. 177-182, 2002.

[9] M. Kimoto, M. Endo, T. Mitsui, T. Okuni, I. Hirao, and S. Yokoyama, "Site-specific incorporation of a photocrosslinking component into RNA by T7 transcription mediated by unnatural base pairs," Chemistry and Biology, vol. 11, no. 1, pp. 47-55, 2004.

[10] R. Kawai, M. Kimoto, S. Ikeda et al., "Site-specific fluorescent labeling of RNA molecules by specific transcription using unnatural base pairs," Journal of the American Chemical Society, vol. 127, no. 49, pp. 17286-17295, 2005.

[11] K. Moriyama, M. Kimoto, T. Mitsui, S. Yokoyama, and I. Hirao, "Site-specific biotinylation of RNA molecules by transcription using unnatural base pairs," Nucleic Acids Research, vol. 33, no. 15, article e129, 2005.

[12] M. Kimoto, T. Mitsui, Y. Harada, A. Sato, S. Yokoyama, and I. Hirao, "Fluorescent probing for RNA molecules by an unnatural base-pair system," Nucleic Acids Research, vol. 35, no. 16, pp. 5360-5369, 2007.

[13] Y. Hikida, M. Kimoto, S. Yokoyama, and I. Hirao, "Site-specific fluorescent probing of RNA molecules by unnatural basepair transcription for local structural conformation analysis," Nature Protocols, vol. 5, no. 7, pp. 1312-1323, 2010.

[14] D. A. Malyshev, J. S. Young, P. Ordoukhanian, and F. E. Romesberg, "PCR with an expanded genetic alphabet," Journal of the American Chemical Society, vol. 131, no. 41, pp. 14620$14621,2009$.

[15] Y. J. Seo, S. Matsuda, and F. E. Romesberg, "Transcription of an expanded genetic alphabet," Journal of the American Chemical Society, vol. 131, no. 14, pp. 5046-5047, 2009.

[16] Y. J. Seo, D. A. Malyshev, T. Lavergne, P. Ordoukhanian, and F. E. Romesberg, "Site-specific labeling of DNA and RNA using an efficiently replicated and transcribed class of unnatural base pairs," Journal of the American Chemical Society, vol. 133, no. 49, pp. 19878-19888, 2011.

[17] I. Hirao, M. Kimoto, T. Mitsui et al., "An unnatural hydrophobic base pair system: site-specific incorporation of nucleotide analogs into DNA and RNA," Nature Methods, vol. 3, no. 9, pp. 729-735, 2006.

[18] M. Morohashi, M. Kimoto, A. Sato, R. Kawai, and I. Hirao, "Site-specific incorporation of functional components into RNA by an unnatural base pair transcription system," Molecules, vol. 17, no. 3, pp. 2855-2876, 2012.

[19] M. Kimoto, R. Kawai, T. Mitsui, S. Yokoyama, and I. Hirao, "An unnatural base pair system for efficient PCR amplification and functionalization of DNA molecules," Nucleic Acids Research, vol. 37, no. 2, article e14, 2009. 
[20] R. Yamashige, M. Kimoto, Y. Takezawa et al., "Highly specific unnatural base pair systems as a third base pair for PCR amplification," Nucleic Acids Research, vol. 40, no. 6, pp. 2793 2806, 2012.

[21] E. Burke and S. Barik, "Megaprimer PCR: application in mutagenesis and gene fusion," Methods in Molecular Biology, vol. 226, pp. 525-532, 2003. 

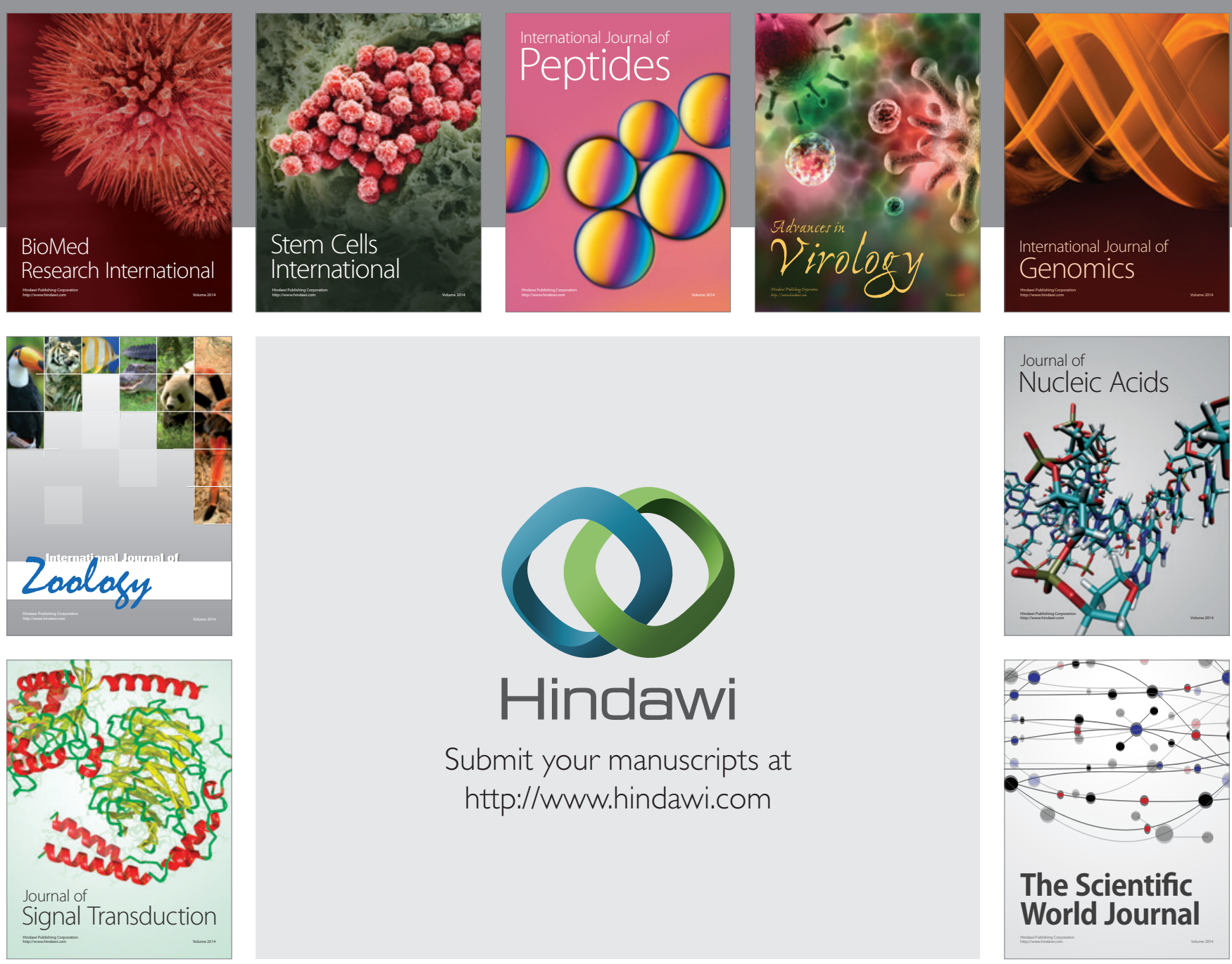

Submit your manuscripts at

http://www.hindawi.com
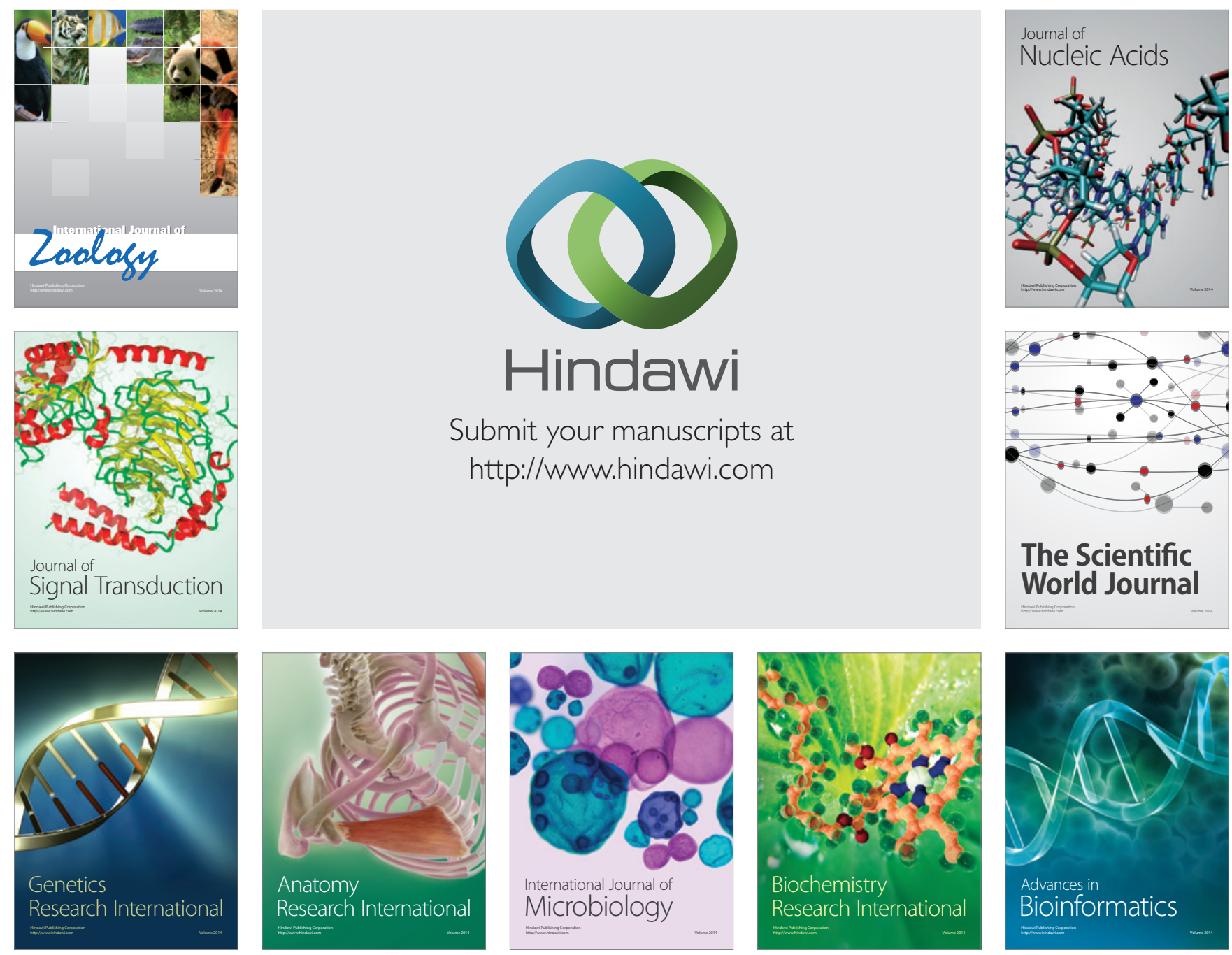

The Scientific World Journal
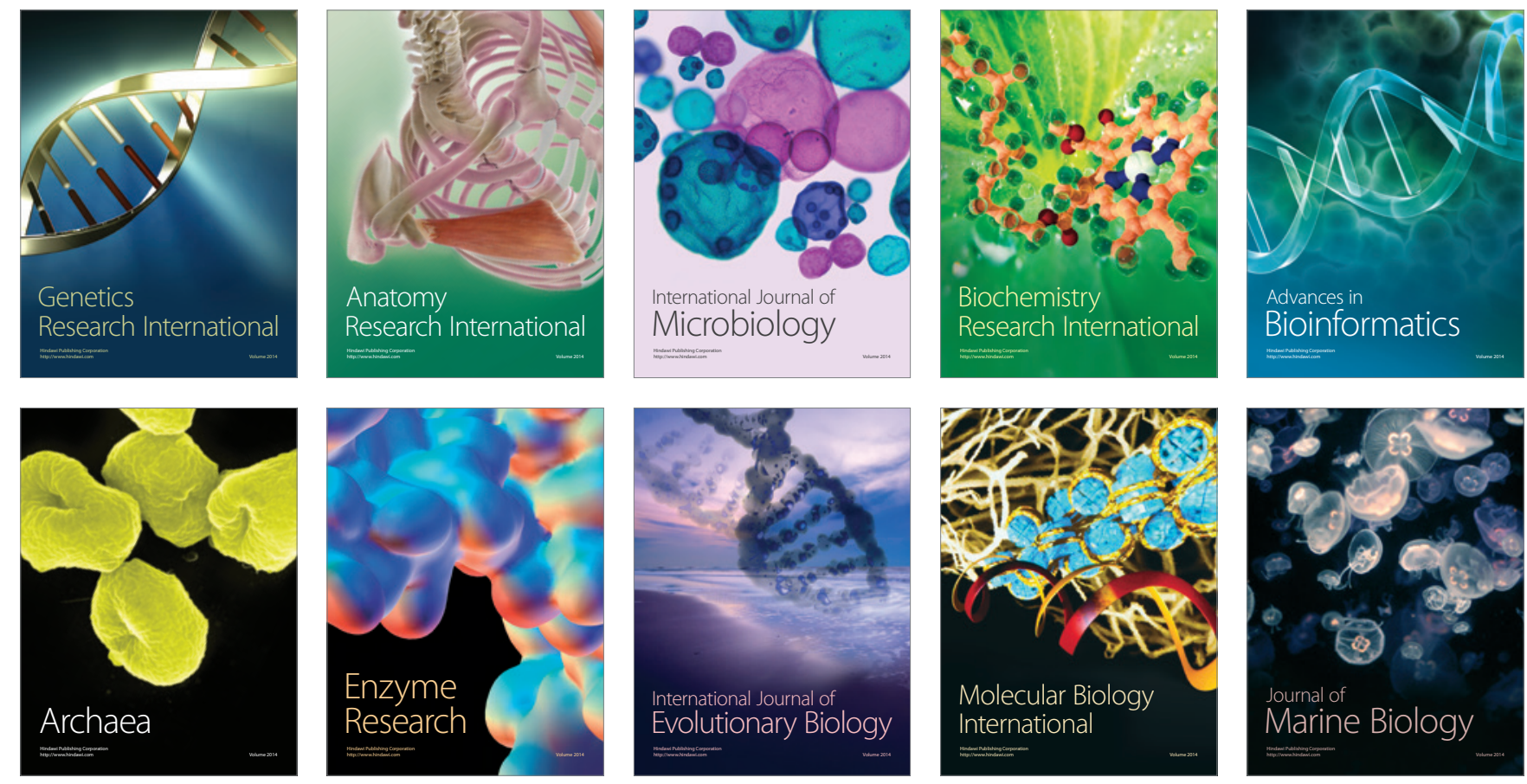